\title{
Oral Health Status of Hearing-Impaired Studer
Attending Special Need Schools in Amhara Region, Ethiopia: A Cross-Sectional Study
}

\author{
Amare Teshome Tefera (ID)', Biruk Girma', Aynishet Adane², Abebe Muche ${ }^{3}$, Tadesse Awoke Ayele ${ }^{4}$, \\ Kefyalew Ayalew Getahun $\mathbb{D}^{5}$, Zelallem Aniley', Semira Ali ${ }^{6}$, Simegnew Handebo $\mathbb{D}^{7}$ \\ 'Department of Dentistry, School of Medicine, College of Medicine and Health Sciences, University of Gondar, Gondar, Ethiopia; ${ }^{2}$ Department of \\ Internal Medicine, School of Medicine, College of Medicine and Health Sciences, University of Gondar, Gondar, Ethiopia; ${ }^{3}$ Department of Anatomy, \\ School of Medicine, College of Medicine and Health Sciences, University of Gondar, Gondar, Ethiopia; ${ }^{4}$ Department of Biostatistics and Epidemiology, \\ Institute of Public health, College of Medicine and Health Sciences, University of Gondar, Gondar, Ethiopia; ${ }^{5}$ Department of Pharmacology, School of \\ Pharmacy, College of Medicine and Health Sciences, University of Gondar, Gondar, Ethiopia; ${ }^{6}$ Department of special need and inclusive education, \\ College of Education, University of Gondar, Gondar, Ethiopia; ${ }^{7}$ Department of health education and Behavioral Sciences, Institute of Public health, \\ College of Medicine and Health Sciences, University of Gondar, Gondar, Ethiopia
}

Correspondence: Amare Teshome Tefera, Department of Dentistry, School of Medicine, College of Medicine and Health Science, University of Gondar, P.O.Box 196, Gondar, Ethiopia, Tel +25I-910517002, Email teferaden@gmail.com

Background: Oral health care is the most common unmet need among individuals with a disability. Individuals with a disability may have compromised oral health needs due to neglect from parents, socioeconomic problems, and communication barriers. In Ethiopia, there is a lack of data on oral health status of the hearing-impaired population. Therefore, this study aimed to assess the oral health status of hearing-impaired students in the Amhara region, Ethiopia.

Methods: A cross-sectional study was conducted from November 2020 to April 2021 on hearing-impaired students in Amhara region, Ethiopia. Data were collected using a pretested interview administered questionnaire and clinical examination. Oral cavity was evaluated using the simplified oral hygiene index, decayed, missed and filled teeth, and community periodontal index. Data analysis was done using SPSS 26.0, and logistic regression analysis was done to identify the risk factors of dental caries and periodontal disease.

Results: A total of 149 hearing impaired students with an age range of 7-30 years were involved in the study. The prevalence of periodontal disease and dental caries was $22.8 \%$ (95\% CI: 16.8, 30.4) and 38.9\% (95\% CI; 32.2, 46.9), respectively. Being grade 1-4 student $(\mathrm{AOR}=3.94,95 \%: 1.16,13.38)$, lack of formal education $(\mathrm{AOR}=4.98,95 \% \mathrm{CI}: 1.00,24.65)$, dental caries $(\mathrm{AOR}=2.51$, $95 \%$ CI: $1.08,5.08)$ and bleeding on probing (AOR $=9.98,95 \%$ CI: 3.69, 26.64) were statistically significant with periodontal disease. Grade level, parents' support during brushing, oral health status, and medication intake were independent factors for dental caries. Conclusion: In the present study, a significant number of hearing-impaired students had periodontal disease and dental caries. School oral health programs and caregivers assisted oral hygiene practices are essential to combat oral health problems in hearing-impaired students. Moreover, a nationwide prospective study with a large sample size will be required to reflect the oral health status of hearingimpaired individuals in the country.

Keywords: oral health status, hearing impairment, dental caries, periodontal disease, DMFT

\section{Background}

In the world, more than 360 million population live with hearing impairment, and $9 \%$ of them are under 15 years. In subSaharan Africa 6 per 1000, live births were hearing impaired, which was high compared with one per 1000 in-developed countries. ${ }^{1,2}$ Hearing impairment was quite common in South Asia, Asia Pacific, and sub-Saharan Africa. ${ }^{3}$ In Ethiopia, it is estimated that $71.2 \%$ of the population with disability were hearing-impaired individuals. ${ }^{1}$ A survey in North West Ethiopia showed that $8.3 \%$ of the study participants had a hearing impairment. ${ }^{4}$ 
Oral health care is the most common unmet need among individuals with disabilities. Health care access was continued to be a challenge for individuals with special needs in the 21 st century. ${ }^{5}$ Individuals with disabilities may have a compromised oral health need due to neglect from parents, socioeconomic problems, and communication barriers. ${ }^{6}$ The high prevalence of oral health problems in individuals with a disability has been attributed to poor oral hygiene practice, lack of awareness about oral hygiene, diet, physical limitations, professional's attitude, and lack of access to oral health care. ${ }^{7}$

Hearing impairment is a disability that can limit one's ability to acquire information and have a negative impact on one's personal health. ${ }^{8}$ The severity and magnitude of dental problems in the hearing impaired population are worse and have more untreated dental conditions than the general people. ${ }^{9}$ The oral hygiene status of children and young adults with hearing disabilities was significantly associated with age, economic status and educational level of parents, especially maternal education. ${ }^{10}$ In India, $65 \%$ of special need school students had dental caries with a mean level of caries prevalence (DMFT) of $1.6 \pm 1.3 .{ }^{11}$ Azfar et al, in Pakistan, reported that $51 \%$ of disabled students had dental caries with a mean DMFT score of $2.08( \pm 2.97)$, and $48.1 \%$ of them had good oral hygiene status $(48.1 \%) .{ }^{12}$

Oral health has a strong psychological, biological, and social consequences as it affects the communication, aesthetics, and quality of life of the affected individuals. ${ }^{13}$ Hearing impaired people had difficulty communicating with health care professionals, and as a result of the communication gap, most dental and medical professionals ignored those patients with hearing impairment. ${ }^{14}$ Assessing the oral health status of hearing-impaired students may reduce future treatment needs, reduce treatment cost and plan preventive and curative measures. ${ }^{14,15}$ Besides, it is also important for resource planning and designing community service for disabled populations based on their needs.

In Ethiopia, some studies were carried out on the oral health status of school children. ${ }^{16-19}$ However, there is no evidence of the oral health status of the hearing-impaired population in the country. Hence, this study aimed to assess the oral health status of hearing-impaired students attending special need schools in Amhara region, Ethiopia.

\section{Methods}

\section{Study Area and Period}

This study was conducted on eight special need schools (Gondar, Bahir Dar, Dessie, and Debre Markos, two from each) in Amhara regional state, Ethiopia. The list of students was taken from their roster. The study was carried out over six months, from November 2020 to April 2021.

\section{Study Design}

School-based cross-sectional study was employed among hearing-impaired school students in Amhara region, Ethiopia.

\section{Inclusion and Exclusion Criteria}

Source and study populations: All hearing-impaired individuals in Amhara regional state were the source population for this study, while hearing-impaired students that attend special need schools were the study population.

Exclusion criteria: Study participants with dental/medical emergency, uncooperative, and who were absent from school during the data collection period were excluded from the study.

\section{Sample Size Determination and Sampling Techniques}

The sample size was determined using a single population proportion formula for another study published to BMC oral health journal entitled "Dental health problems and treatment-seeking behavior among special need school students in Amhara Region", ${ }^{20}$ where the following assumptions were considered: P (50\% proportion of dental health problem among special need student), $\mathrm{d}$ (Margin of error: $5 \%$ ), $\mathrm{Z} \alpha / 2$ (the value of the standard normal curve score corresponding to the given confidence interval $=1.96$ ) corresponding to $95 \%$ confidence level, and $15 \%$ non-response rate. In this study, we have included all (149) hearing-impaired special needs students. 


\section{Operational Definition}

Dental caries: A participant was considered to have a dental caries if he/she had decayed, teeth, missed teeth due to dental caries, and restored teeth in both primary and permanent dentitions.

Hearing Impairment: The term hearing impairment refers to students who had both complete and partial hearing problem and attend special need schools in the study area.

Periodontal disease: Periodontal diseases are infections and inflammation of the gum and bone that surround and support the teeth. In the present study, a student is considered to have periodontal disease if and only if the participant had a periodontal pocket depth of greater than $3 \mathrm{~mm}$.

Oral health status: The oral hygiene of the students was evaluated using the simplified oral hygiene index. ${ }^{21}$

\section{Study Variables}

The dependent variables in this study were dental caries, periodontal disease, and oral health status. Whereas sociodemographic characteristics, tooth brushing habits, and dietary habits were independent variables.

\section{Data Collection Tools and Techniques}

The data collection tool had two parts. The first part includes a structured questionnaire that targets to record the sociodemographic, tooth brushing habits and dietary habits. The second part includes an intra-oral examination.

\section{Questionnaire Administered Interview}

A structured pretested interview administered questionnaire was used to collect data on sociodemographic, oral health behavior, medical condition, duration of hearing impairment, and oral habits (pen/pencil/finger sucking and mouth breathing pattern). The questionnaire was adopted from the WHO Oral Health Survey tool ${ }^{14}$ and different kinds of the literature. ${ }^{22,23}$ The tool was prepared in English and translated into the local language-Amharic. It was then translated back into English by two non-dental professionals, and the translated and original questionnaire was assessed if they achieve conceptual and semantic equivalence, and they did not require any further amendment. Special need experts that knew sign language were involved in communicating the information between the students and dental professionals.

\section{Intra-Oral Examination}

The oral cavity of the study participants was evaluated using a community periodontal index (CPI) probe, mouth mirror, and dental explorers as WHO criteria to diagnose periodontal disease and dental caries. Four examiners, who were calibrated before the actual study to reduce inter-examiner variation, were involved in the intra-oral examination process. Moreover, 2 special need experts were involved in the process. The data recorded were DMFT index (decayed, missed, and filled teeth), oral hygiene status (based on simplified oral hygiene index, OHI-S) ${ }^{21}$ and periodontal status using the community periodontal index (CPI). The OHI-S was recorded on six surfaces of index teeth (buccal surface of 11, 16, 26, and 31) and lingual surface of 36 and 46. For under 18 years of age children, a guideline that was developed by the British Society of Periodontology and the British Society of Pediatric Dentistry "Guidelines for Periodontal Screening and Management of Children and Adolescents Under 18 Years of Age" was used. ${ }^{24}$ Students with a dental emergency were referred to the nearby dental care centers for treatment. The intra-oral examination was done at the schools using normal light/daylight, tongue depressor, mouth mirror, periodontal probe, and dental explorers. A pilot test was done on 15 hearing impaired students at Injibara in October 2020. Cohen kappa coefficient was then calculated for inter-examiner reliability, and ranged from $0.88-0.95$, indicating good and almost perfect results. To avoid cross-infection of COVID-19, maximum preventive methods and strategies were implemented. A five-day training was given for the data collectors. Moreover, there was daily onsite supervision to check the completeness of the questionnaire.

\section{Data Analysis}

The statistical package for social science, version 26.0 (IBM Corp., Armonk, NY, USA), was used for the statistical analysis. Descriptive data of frequency, percentage, mean, and SD were calculated and presented in terms of tables. A bivariable and multivariable logistic regression model was used to identify the factors associated with dental caries and 
periodontal disease among hearing-impaired students in the Amhara region. Study variables with $\mathrm{P}<0.05$ at a $95 \%$ confidence interval were considered statistically significant.

\section{Ethical Consideration}

The ethical clearance was obtained from the University of Gondar Institutional Review Board (Ref. No: V/P/RCS/05/ 541/2020). Furthermore, permission was requested and obtained from each town's education Bureau and special need schools. Written informed consent was obtained from parents or legal guardians, and assent from the students themselves was obtained before the study. The study was done in accordance with the declaration of Helsinki (Code of Ethics of the World Medical Association).

\section{Results}

\section{Demographic Characteristics}

A total of 149 hearing impaired students of 7-30 years and a mean age of 15.51 ( $\mathrm{SD} \pm 3.904)$ were involved in the study. The majority of the study participants were females (57\%) and 13-18 years old (65.8\%). Almost half of the students were grade 5 to grade $8(48.3 \%)$. Most of the study participants (84.6\%) had a family monthly income of $\leq 2500$ Ethiopian Birr (Table 1).

\section{Oral Health Behaviors of the Study Participants}

One hundred forty-two students (95.3\%) had a habit of sugary food intake, of this $30.2 \%$ took twice, or more per day. More than 3/4th (80.5\%) of the participants had tooth brushing habits. Of this, only $7.4 \%$ of them brushed their teeth twice a day, and $20.1 \%$ of the study participants had parental support during tooth brushing. Tooth stick was the commonly (45.0\%) used tooth-brushing material. About 17 (11.4\%) students had other medical comorbidities in addition to their hearing disability. Regarding the oral habits of the study participants, $36.2 \%$ were mouth breathers and $17.4 \%$ had sucking habits (finger, pen, pencil, etc.) (Table 2).

\section{Oral Health Status}

The oral health status of the participants was evaluated using the simplified oral hygiene index, and was $36.2 \%, 32.2 \%$, and $31.5 \%$ for Good, Fair, and poor oral hygiene, respectively.

\section{Prevalence of Periodontal Disease}

The prevalence of periodontal disease among hearing-impaired students in the Amhara regional state was 22.8\% (95\% CI: $16.8,30.4)$. Nearly one-third $(29.2 \%)$ of students above the age of 18 years had periodontal disease. High prevalence was reported among 18 and above years (29.2\%), grade 9-12 students (35.0\%), and students with poor oral health status (59.6\%), class-II malocclusion (45.5\%), participants who had no family support during tooth brushing (25.0\%), and dental caries (32.8\%). Moreover, 43.6\% (95\% CI: 35.6, 51.2) of the study participants had bleeding on probing (BOP). Twelve (27.9\%) students with malocclusion had periodontal disease (Table 3).

\section{Prevalence of Dental Caries}

The prevalence of dental caries among hearing-impaired school students in the region was $38.9 \%(32.2,46.9)$. Forty-one (41.8\%) of students aged 13 to 18 years had dental caries, and $64.7 \%$ of students with comorbidities had dental caries. Moreover, the prevalence was high in students with class-II malocclusion (50.0\%), periodontal disease (55.9\%), and sucking habit (53.8\%). About $60 \%$ of students with family support during their oral hygiene practice had dental caries. Students with a history of medication intake had a higher prevalence of dental caries than those who were not on medication (78.6\% vs $34.8 \%)$ (Table 4$)$. 
Table I Sociodemographic Characteristics of Hearing-Impaired Students Attending Special Need Schools in the Amhara Region, Ethiopia

\begin{tabular}{|c|c|c|c|}
\hline \multicolumn{2}{|l|}{ Variables } & \multirow{2}{*}{$\begin{array}{l}\text { Frequency (n) } \\
64\end{array}$} & \multirow{2}{*}{$\begin{array}{l}\text { Percentage (\%) } \\
43.0\end{array}$} \\
\hline Sex & Male & & \\
\hline & Female & 85 & 57.0 \\
\hline \multirow[t]{3}{*}{ Age } & $7-12$ years & 27 & 18.1 \\
\hline & $13-18$ years & 98 & 65.8 \\
\hline & $>18$ years & 24 & 16.1 \\
\hline \multirow[t]{4}{*}{ Religion } & Orthodox & 107 & 71.8 \\
\hline & Catholic & 10 & 6.7 \\
\hline & Muslim & 29 & 19.5 \\
\hline & Protestant & 3 & 2.0 \\
\hline \multirow[t]{4}{*}{ Location of the participants } & Gondar & 39 & 26.2 \\
\hline & Bahir Dar & 39 & 26.2 \\
\hline & Debre Markos & 27 & 18.1 \\
\hline & Dessie & 44 & 29.5 \\
\hline \multirow[t]{3}{*}{ Grade level } & $\mathrm{I}-4$ & 57 & 38.3 \\
\hline & $5-8$ & 72 & 48.3 \\
\hline & $9-12$ & 20 & 13.4 \\
\hline \multirow[t]{3}{*}{ Mothers educational status } & No formal education & 126 & 89.4 \\
\hline & Primary & 6 & 4.3 \\
\hline & Secondary and above & 9 & 6.4 \\
\hline \multirow[t]{3}{*}{ Fathers educational status } & No formal education & 116 & 82.9 \\
\hline & Primary & 7 & 5.0 \\
\hline & Secondary and above & 17 & 12.1 \\
\hline \multirow[t]{2}{*}{ Family monthly income } & $<2500$ Ethiopian birr & 115 & 84.6 \\
\hline & $\geq 2500$ Ethiopian Birr & 21 & 15.4 \\
\hline
\end{tabular}

\section{The Decayed Missed and Filled Teeth (DMFT/dmfts)}

The mean DMFT score was $1.15 \pm 1.654$. Decay was the highest component of the mean DMFT, with a mean value of $0.77 \pm 1.258$. There was no filed tooth among the study participants. The mean DMFT was $1.54 \pm 1.911$ for those above 18 years and $1.90 \pm 1.917$ for those grades $9-12$ students. The mean dmft in the primary dentition was $0.11 \pm 0.564$ (Table 5).

\section{Factors Associated with Periodontal Disease}

As shown in Table 6, variables with a p-value of $<0.25$ in bivariate logistic regression model such as: age, sex, grade level, sugary food intake, mouth breathing, parents support during tooth brushing, oral health status, paternal educational status, class-II malocclusion, bleeding on probing, medication intake and dental caries were entered into the multivariate 
Table 2 Oral Health Behavior of Hearing-Impaired Students Attending Special Need Schools in Amhara Region, Ethiopia, 2020/21 ( $n=149)$

\begin{tabular}{|c|c|c|c|}
\hline \multicolumn{2}{|l|}{ Variables } & \multirow{2}{*}{$\begin{array}{l}\text { Frequency } \\
142\end{array}$} & \multirow{2}{*}{$\begin{array}{l}\text { Percentage } \\
95.3\end{array}$} \\
\hline Sugary food intake & Yes & & \\
\hline & No & 7 & 4.7 \\
\hline \multirow[t]{4}{*}{ Frequency of sugary food intake } & Some times & 63 & 42.3 \\
\hline & Once a day & 34 & 22.8 \\
\hline & Twice a day & 38 & 25.5 \\
\hline & Three and more times a day & 7 & 4.7 \\
\hline \multirow[t]{6}{*}{ Type of sugary food intake } & Milk with sugar & 63 & 42.3 \\
\hline & Juice & 46 & 30.9 \\
\hline & Chewing gum & 70 & 47.0 \\
\hline & Soft drinks & 57 & 38.3 \\
\hline & Deserts & 70 & 47.0 \\
\hline & Candy & 85 & 57.0 \\
\hline \multirow[t]{2}{*}{ Tooth brushing } & Yes & 120 & 80.5 \\
\hline & No & 29 & 19.5 \\
\hline \multirow[t]{4}{*}{ Frequency of tooth brushing } & Some times & 53 & 35.6 \\
\hline & 2-3 times per week & 9 & 6.0 \\
\hline & Once a day & 47 & 31.5 \\
\hline & Twice a day & 11 & 7.4 \\
\hline \multirow[t]{4}{*}{ Tooth brushing materials } & Toothbrush & 51 & 34.2 \\
\hline & Tooth stick* & 67 & 45.0 \\
\hline & Charcoal & I & 0.7 \\
\hline & Other & I & 0.7 \\
\hline \multirow{2}{*}{$\begin{array}{l}\text { Parents support during tooth } \\
\text { brushing }\end{array}$} & Yes & 30 & 20.1 \\
\hline & No & 119 & 79.9 \\
\hline \multirow[t]{4}{*}{ Time of tooth brushing } & Before bed & 15 & 10.1 \\
\hline & Before breakfast & 81 & 54.4 \\
\hline & After breakfast and before bed & 19 & 12.8 \\
\hline & At school & I & 0.7 \\
\hline \multirow[t]{3}{*}{ Brushing after meal } & Never & 27 & 18.1 \\
\hline & Some times & 82 & 55.0 \\
\hline & Always & 7 & 4.7 \\
\hline
\end{tabular}

(Continued) 
Table 2 (Continued).

\begin{tabular}{|l|l|l|l|}
\hline \multicolumn{2}{|l|}{ Variables } & Frequency & Percentage \\
\hline \multirow{4}{*}{ Changing toothbrush } & Monthly & 14 & 9.4 \\
\cline { 2 - 4 } & Every three months & 23 & 15.4 \\
\cline { 2 - 4 } & Every six months & 24 & 16.1 \\
\cline { 2 - 4 } & Yearly & 24 & 16.1 \\
\hline \multirow{3}{*}{ Mouth breathing* } & Yes & 54 & 36.2 \\
\cline { 2 - 4 } & No & 95 & 63.8 \\
\hline \multirow{3}{*}{ Sucking habit } & Yes & 26 & 17.4 \\
\cline { 2 - 4 } & No & 123 & 82.6 \\
\hline Comorbidity & Yes & 17 & 11.4 \\
\cline { 2 - 4 } & No & 132 & 88.6 \\
\hline Medication & Yes & 14 & 9.4 \\
\cline { 2 - 4 } & No & 135 & 90.6 \\
\hline
\end{tabular}

Notes: *The breathing pattern is assessed using the butterfly test. *Tooth Stick: a traditional tooth brushing material that is prepared from Stick.

logistic regression model. In the multivariable logistic regression analysis, grade level, fathers' educational status, dental caries, and bleeding on probing were statistically significant factors for periodontal disease.

Hearing-impaired students attending grades $1-4(\mathrm{AOR}=3.94(95 \% \mathrm{CI} ; 1.159,13.379))$ were 3.94 times more likely to have periodontal disease than students attending grades 5-8 and 9-12. Students that had illiterate fathers were 4.98 times more likely to have periodontal disease $(\mathrm{AOR}=4.98,95 \% \mathrm{CI} ; 1.00,24.65)$. Students with dental caries were 2.51 times more likely to have periodontal disease than students who do not have dental caries (AOR $=2.51,95 \%$ CI: 1.08 , 5.08). Special needs school students with bleeding on probing were 9.89 times more likely to have periodontal disease than normal students were (AOR $=9.89,95 \% \mathrm{CI} ; 3.69,26.64)$ (Table 6).

\section{Factors Associated with Dental Caries}

In the bivariate logistic regression model, grade level, parents' support during tooth brushing, comorbidity, oral health status, class-II malocclusion, sucking habits, periodontal disease, and medication intake had a p-value of $<0.25$. These variables were entered into the multivariable logistic regression model, and grade level, parents' support during tooth brushing, oral health status, and medication intake were significantly associated with dental caries.

Grade 9-12 students (AOR $=4.45$ (95\% CI: 1.10, 21.28) were 4.45 times more likely to have dental caries than students attending grade 1-4 and grade 5-8. Students who had no family support during tooth brushing were 5.10 times more likely to have dental caries than those who had family support during their oral hygiene practice (AOR $=5.10,95 \%$ CI: $1.89,13.76)$. Students with poor oral health status were 8.07 times more likely to have dental caries compared to good oral health status students $(\mathrm{AOR}=8.07$ (95\% CI; 2.49, 26.18). Moreover, students who were on medication were 9.26 times more likely to have dental caries than students who did not take medications for systemic disease (AOR $=$ 9.26; 95\% CI: 2.17, 39.45) (Table 7).

\section{Discussion}

In this study, the prevalence of periodontal disease was $22.8 \%$ (95\% CI: $16.8,30.4)$. The study found a significant association between grade level, fathers' educational status, dental caries, and bleeding on probing with periodontal disease. About 38.9\% (95\% CI; 32.2, 46.9) of the study participants had dental caries, and grade level, parents support 
Table 3 Prevalence of Periodontal Disease Among Hearing-Impaired School Students in Amhara Regional State, Ethiopia, 2020/2I

\begin{tabular}{|c|c|c|c|c|}
\hline \multicolumn{2}{|l|}{ Variables } & \multicolumn{2}{|c|}{ Periodontal Disease } & \multirow[t]{2}{*}{ P-value (Chi-Square) } \\
\hline & & Yes & No & \\
\hline \multirow[t]{3}{*}{ Age } & $7-12$ years & I (3.7\%) & $26(96.3 \%)$ & \multirow[t]{3}{*}{0.031} \\
\hline & $13-18$ years & $26(26.5 \%)$ & 72 (73.5\%) & \\
\hline & Above 18 years & 7 (29.2\%) & 17 (70.8\%) & \\
\hline \multirow[t]{2}{*}{ Sex } & Male & $18(28.1 \%)$ & 46 (7I.9\%) & \multirow[t]{2}{*}{0.180} \\
\hline & Females & $16(18.8 \%)$ & 69 (81.2\%) & \\
\hline \multirow[t]{4}{*}{ Location } & Gondar & II (28.2\%) & 28 (7I.8\%) & \multirow[t]{4}{*}{0.576} \\
\hline & Bahir Dar & $9(23.1 \%)$ & $30(76.9 \%)$ & \\
\hline & Debre Markos & 7 (25.9\%) & 20 (74.1\%) & \\
\hline & Dessie & 7 (I5.9\%) & 37 (84.l\%) & \\
\hline \multirow[t]{3}{*}{ Grade level } & $1-4$ & 7 (I2.3\%) & $50(87.7 \%)$ & \multirow[t]{3}{*}{0.043} \\
\hline & $5-8$ & $20(27.8 \%)$ & 52 (72.2\%) & \\
\hline & $9-12$ & 7 (35.0\%) & $13(65.0 \%)$ & \\
\hline \multirow[t]{4}{*}{ Religion } & Orthodox & $25(23.4 \%)$ & $82(76.6 \%)$ & \multirow[t]{4}{*}{0.955} \\
\hline & Catholic & $2(20.0 \%)$ & $8(80.0 \%)$ & \\
\hline & Muslim & $6(20.7 \%)$ & $23(79.3 \%)$ & \\
\hline & Protestant & I (33.3\%) & $2(66.7 \%)$ & \\
\hline \multirow[t]{2}{*}{ Sugary food intake } & Yes & 31 (2I.8\%) & III (78.2\%) & \multirow[t]{2}{*}{0.196} \\
\hline & No & $3(42.9 \%)$ & $4(57.1 \%)$ & \\
\hline \multirow[t]{2}{*}{ Tooth brushing } & Yes & 27 (22.5\%) & 93 (77.5\%) & \multirow[t]{2}{*}{0.850} \\
\hline & No & 7 (24.1\%) & 22 (75.9\%) & \\
\hline \multirow[t]{4}{*}{ Frequency of tooth brushing } & Some times & $13(24.5 \%)$ & 40 (75.5\%) & \multirow[t]{4}{*}{0.963} \\
\hline & 2-3 times per week & $2(22.2 \%)$ & 7 (77.8\%) & \\
\hline & Once a day & 9 (19.1\%) & 38 (80.9\%) & \\
\hline & Twice a day & $3(27.3 \%)$ & 8 (72.7\%) & \\
\hline \multirow{2}{*}{$\begin{array}{l}\text { Parents support during } \\
\text { tooth brushing }\end{array}$} & Yes & $4(13.3 \%)$ & $26(86.7 \%)$ & \multirow[t]{2}{*}{0.183} \\
\hline & No & $22(25.0 \%)$ & 66 (75.0\%) & \\
\hline \multirow[t]{2}{*}{ Mouth breathing } & Yes & $6(11.1 \%)$ & 48 (88.9\%) & \multirow[t]{2}{*}{0.010} \\
\hline & No & 28 (29.5\%) & 67 (70.5\%) & \\
\hline \multirow[t]{2}{*}{ Comorbidity } & Yes & $3(17.6 \%)$ & 14 (82.4\%) & \multirow[t]{2}{*}{0.589} \\
\hline & No & 31 (23.5\%) & 101 (76.5\%) & \\
\hline \multirow[t]{2}{*}{ Medication } & Yes & 3 (21.4\%) & II (78.6\%) & \multirow[t]{2}{*}{0.896} \\
\hline & No & 31 (23.0\%) & 104 (77.0\%) & \\
\hline
\end{tabular}


Table 3 (Continued).

\begin{tabular}{|c|c|c|c|c|}
\hline \multicolumn{2}{|l|}{ Variables } & \multicolumn{2}{|c|}{ Periodontal Disease } & \multirow[t]{2}{*}{ P-value (Chi-Square) } \\
\hline & & Yes & No & \\
\hline \multirow[t]{3}{*}{ Oral health status } & Good & $0(0.0 \%)$ & $54(100.0 \%)$ & \multirow[t]{3}{*}{$<0.001$} \\
\hline & Fair & $6(12.5 \%)$ & $42(87.5 \%)$ & \\
\hline & Poor & $28(59.6 \%)$ & $19(40.4 \%)$ & \\
\hline \multirow[t]{3}{*}{ Mothers educational status } & No formal education & 30 (23.8\%) & 96 (76.2\%) & \multirow[t]{3}{*}{$0.98 \mathrm{I}$} \\
\hline & Primary education & I (I6.7\%) & $5(83.3 \%)$ & \\
\hline & High school and above & $2(22.2 \%)$ & 7 (77.8\%) & \\
\hline \multirow[t]{3}{*}{ Fathers educational status } & No formal education & $23(19.8 \%)$ & $93(80.2 \%)$ & \multirow[t]{3}{*}{0.327} \\
\hline & Primary education & $2(28.6 \%)$ & 5 (7I.4\%) & \\
\hline & High school and above & $6(35.3 \%)$ & II (64.7\%) & \\
\hline \multirow[t]{2}{*}{ Class-I occlusion } & Yes & $22(20.8 \%)$ & $84(79.2 \%)$ & \multirow[t]{2}{*}{0.346} \\
\hline & No & $12(27.9 \%)$ & 31 (72.1\%) & \\
\hline \multirow[t]{2}{*}{ Class-II malocclusion } & Yes & $10(45.5 \%)$ & $12(54.5 \%)$ & \multirow[t]{2}{*}{0.006} \\
\hline & No & 24 (I8.9\%) & $103(81.1 \%)$ & \\
\hline \multirow[t]{2}{*}{ Bleeding on probing } & Yes & $28(43.1 \%)$ & 37 (56.9\%) & \multirow[t]{2}{*}{$<0.001$} \\
\hline & No & $6(7.1 \%)$ & 78 (92.9\%) & \\
\hline \multirow[t]{2}{*}{ Dental caries } & Yes & 19 (32.8\%) & 39 (67.2\%) & \multirow[t]{2}{*}{0.018} \\
\hline & No & $15(16.5 \%)$ & $76(83.5 \%)$ & \\
\hline \multirow[t]{2}{*}{ Malocclusion } & Yes & $12(27.9 \%)$ & 31 (72.1\%) & \multirow[t]{2}{*}{0.346} \\
\hline & No & $22(20.8 \%)$ & 84 (79.2\%) & \\
\hline
\end{tabular}

during tooth brushing, oral health status, and medication intake were independent factors for dental caries among hearing-impaired students.

When compared with the present study, a low prevalence (7\%) of periodontal disease (pocket depth of $>3 \mathrm{~mm}$ ) was reported in India in 2013 among the special needs school students. ${ }^{25}$ Likewise, Ameer and collaborators found a low prevalence $(9.1 \%)$ of periodontal disease among hearing-impaired individuals. ${ }^{26} \mathrm{~A}$ high prevalence of periodontal disease might be due to an individual's low awareness about oral hygiene, communication problem, and lack of oral health care. ${ }^{26,27}$ Presumably, it may also be due to financial problems to purchase tooth brushing materials as most of the participants were from a low monthly income family and lack of parents' support during tooth brushing.

The current study found that $7.4 \%$ of the hearing-impaired students brushed their teeth at least twice a day. A high frequency was reported in Pakistan where $28.3 \%$ of hearing-impaired children brushed their teeth twice a day. ${ }^{12}$ Interestingly, Rajabloo et al recently reported that half of (49.2\%) of hearing-impaired special need schools in Iran had a habit of brushing their teeth once daily. ${ }^{28}$ The low oral hygiene practice in our study subjects might be due to socioeconomic problems and/or lack of oral health awareness in our study participants. Our study also found that students who did not have family support while brushing their teeth were 5.1 times more likely to have dental caries than those who did have family support in their daily oral hygiene practice. A study done in India found that supervised oral hygiene 
Table 4 Factors Associated with Dental Caries Among Hearing-Impaired Students in Special Need Schools in Amhara Region, Ethiopia, 2020/21

\begin{tabular}{|c|c|c|c|c|}
\hline \multicolumn{2}{|l|}{ Variables } & \multicolumn{2}{|c|}{ Dental Caries } & \multirow[t]{2}{*}{ p-value (Chi-Square) } \\
\hline & & Yes & No & \\
\hline \multirow[t]{3}{*}{ Age } & $7-12$ years & $8(29.6 \%)$ & 19 (70.4\%) & \multirow[t]{3}{*}{0.509} \\
\hline & $13-18$ years & $4 \mid(4 I .8 \%)$ & 57 (58.2\%) & \\
\hline & Above 18 years & $9(37.5 \%)$ & $15(62.5 \%)$ & \\
\hline \multirow[t]{2}{*}{ Sex } & Male & $24(37.5 \%)$ & $40(62.5 \%)$ & \multirow[t]{2}{*}{0.757} \\
\hline & Females & 34 (40.0\%) & $34(60.0 \%)$ & \\
\hline \multirow[t]{3}{*}{ Grade level } & $1-4$ & $21(36.8 \%)$ & $36(63.2 \%)$ & \multirow[t]{3}{*}{0.112} \\
\hline & $5-8$ & $25(34.7 \%)$ & 47 (65.3\%) & \\
\hline & $9-12$ & $12(60.0 \%)$ & $8(40.0 \%)$ & \\
\hline \multirow[t]{4}{*}{ Religion } & Orthodox & 39 (36.4\%) & $68(63.6 \%)$ & \multirow[t]{4}{*}{0.709} \\
\hline & Catholic & $4(40.0 \%)$ & $6(60.0 \%)$ & \\
\hline & Muslim & $14(48.3 \%)$ & $15(51.7 \%)$ & \\
\hline & Protestant & I (33.3\%) & $2(66.7 \%)$ & \\
\hline \multirow[t]{2}{*}{ Sugary food intake } & Yes & $56(39.4 \%)$ & $86(60.6 \%)$ & \multirow[t]{2}{*}{0.565} \\
\hline & No & $2(71.4 \%)$ & $5(28.6 \%)$ & \\
\hline \multirow[t]{2}{*}{ Tooth brushing } & Yes & 47 (39.2\%) & $73(60.8 \%)$ & \multirow[t]{2}{*}{0.903} \\
\hline & No & II (37.9\%) & $18(62.1 \%)$ & \\
\hline \multirow[t]{4}{*}{ Frequency of tooth brushing } & Some times & $18(34.0 \%)$ & $35(66.0 \%)$ & \multirow[t]{4}{*}{0.749} \\
\hline & 2-3 times per week & $3(33.3 \%)$ & $6(66.7 \%)$ & \\
\hline & Once a day & $22(46.8 \%)$ & $25(53.2 \%)$ & \\
\hline & Twice a day & $4(36.4 \%)$ & $7(63.6 \%)$ & \\
\hline \multirow[t]{2}{*}{ Parents support during tooth brushing } & Yes & $18(60.0 \%)$ & $12(40.0 \%)$ & \multirow[t]{2}{*}{0.006} \\
\hline & No & $28(31.8 \%)$ & $60(68.2 \%)$ & \\
\hline \multirow[t]{2}{*}{ Mouth breathing } & Yes & $22(40.7 \%)$ & $32(59.3 \%)$ & \multirow[t]{2}{*}{0.732} \\
\hline & No & $36(37.9 \%)$ & $59(62.1 \%)$ & \\
\hline \multirow[t]{2}{*}{ Comorbidity } & Yes & II (64.7\%) & $6(35.3 \%)$ & \multirow[t]{2}{*}{0.021} \\
\hline & No & $47(35.6 \%)$ & $85(64.4 \%)$ & \\
\hline \multirow[t]{2}{*}{ Medication } & Yes & II (78.6\%) & $3(21.4 \%)$ & \multirow[t]{2}{*}{0.001} \\
\hline & No & 47 (34.8\%) & $88(65.2 \%)$ & \\
\hline \multirow[t]{3}{*}{ Oral health status } & Good & $14(25.9 \%)$ & $40(74.1 \%)$ & \multirow[t]{3}{*}{0.000} \\
\hline & Fair & $15(31.3 \%)$ & $33(68.8 \%)$ & \\
\hline & Poor & $29(61.7 \%)$ & 18 (38.3\%) & \\
\hline
\end{tabular}

(Continued) 
Table 4 (Continued).

\begin{tabular}{|c|c|c|c|c|}
\hline \multicolumn{2}{|l|}{ Variables } & \multicolumn{2}{|c|}{ Dental Caries } & \multirow[t]{2}{*}{ p-value (Chi-Square) } \\
\hline & & Yes & No & \\
\hline \multirow[t]{3}{*}{ Mothers educational status } & No formal education & $29(40.3 \%)$ & $43(59.7 \%)$ & \multirow[t]{3}{*}{0.304} \\
\hline & Read and Write & $22(40.7 \%)$ & $32(59.3 \%)$ & \\
\hline & Formal education & $3(20.0 \%)$ & $12(80.0 \%)$ & \\
\hline \multirow[t]{3}{*}{ Fathers educational status } & No formal education & $24(37.5 \%)$ & $40(62.5 \%)$ & \multirow[t]{3}{*}{0.838} \\
\hline & Read and Write & $21(40.4 \%)$ & $31(59.6 \%)$ & \\
\hline & Formal education & $8(33.3 \%)$ & $16(66.7 \%)$ & \\
\hline \multirow[t]{2}{*}{ Class-2 malocclusion } & Yes & II (50.0\%) & II (50.0\%) & \multirow[t]{2}{*}{0.249} \\
\hline & No & 47 (37.0\%) & $80(63.0 \%)$ & \\
\hline \multirow[t]{2}{*}{ Bleeding on probing } & Yes & $36(55.4 \%)$ & $29(44.6 \%)$ & \multirow[t]{2}{*}{0.000} \\
\hline & No & $22(26.2 \%)$ & $62(73.8 \%)$ & \\
\hline \multirow[t]{2}{*}{ Sucking habits } & Yes & $14(53.8 \%)$ & $12(46.2 \%)$ & \multirow[t]{2}{*}{0.086} \\
\hline & No & 44 (35.8\%) & 79 (64.2\%) & \\
\hline \multirow[t]{2}{*}{ Periodontal disease } & Ye & $19(55.9 \%)$ & $15(44.1 \%)$ & \multirow[t]{2}{*}{0.021} \\
\hline & No & 39 (33.9\%) & $76(66.1 \%)$ & \\
\hline
\end{tabular}

practice resulted in a marked improvement in oral hygiene and reduced the incidence and severity of oral health problems in hearing-impaired individuals. ${ }^{29}$

Individuals with disabilities have a higher prevalence of dental caries than the non-disabled population. ${ }^{30,31}$ The current study found that $38.9 \%$ (95\% CI: 32.2, 46.9) of the hearing-impaired students had dental caries. Similar results were reported in Brazil ${ }^{32}$ (42.9\%), India (35.32\%), ${ }^{33}$ and Rwanda (42.4\%). ${ }^{34} \mathrm{~A}$ slightly higher prevalence of dental caries was documented in hearing-impaired children in India (56\%), ${ }^{35}$ Thailand (53.6\%), ${ }^{36}$ and China (55.9\%). ${ }^{37}$ Azfar et al reported that over half (51\%) of hearing and speech-impaired children in Pakistan had dental caries. ${ }^{12}$ Moreover, a much higher rate of dental caries was reported among hearing-impaired college students in Thailand $(82.5 \%)^{36}$ and Saudi Arabia $(82.2 \%){ }^{38}$ The difference might be due to socioeconomic variation between the study participants, and the difference in public welfare for the disabled population among countries. ${ }^{39}$

Our study found that $31.5 \%$ of hearing-impaired students had poor oral hygiene status. A slightly higher prevalence $(40 \%)$ of poor oral health status was reported in Iraq among deaf persons. ${ }^{40} \mathrm{~A}$ low prevalence of poor oral health status among hearing-impaired children in Nigeria $(22.2 \%)^{41}$ and Pakistan $(5.7 \%){ }^{12}$ Moreover, the current study found a significant association between poor oral hygiene status and dental caries. Recently, Vichayanrat et al reported that hearing-impaired college students in Thailand with poor oral hygiene status were 3.26 times more likely to have dental caries (OR 3.26; 95\% CI: 1.64-6.45) than those with good oral hygiene status. ${ }^{36}$ Likewise, a cross-sectional study among 226 randomly selected children living with disabilities in Rwanda found that participants with good oral hygiene had low dental caries than those with poor oral hygiene status (AOR $=0.296$, CI: 0.159; 0.550). ${ }^{34}$ The high risk of dental caries in students with poor oral hygiene status might be due to the accumulation of dental plaque, which contains cariogenic bacteria that are involved in the initiation and progression of dental caries. Moreover, limitation in hearing might be contributed to limited understanding, and fear of communicating their oral health needs and procedures. ${ }^{42}$

In the present study, the mean DMFT score among the hearing-impaired school students was $1.15 \pm 1.654$. In addition, the mean $\mathrm{dmft}$ in the primary dentition was $0.11 \pm 0.564$. A slightly higher result was reported in India where 
Table 5 DMFT/dmft Index of Hearing-Impaired Students Attending Special Need Schools in Amhara Region, Ethiopia, 2020/2I

\begin{tabular}{|l|l|l|l|}
\hline \multicolumn{2}{|l|}{ Variables } & $\begin{array}{l}\text { Mean DMFT (Permanent } \\
\text { Dentition) }\end{array}$ & Mean dmft (Primary Dentition) \\
\hline \multirow{3}{*}{ Age } & $7-12$ years & $0.74 \pm 1.831$ & $0.63 \pm 1.214$ \\
\cline { 2 - 4 } & $13-18$ years & $1.16 \pm 1.524$ & $0.00 \pm 0.000$ \\
\cline { 2 - 4 } & Above 18 years & $1.54 \pm 1.911$ & $0.00 \pm 0.000$ \\
\hline \multirow{3}{*}{ Sex } & Male & $1.05 \pm 1.474$ & $0.14 \pm 0.639$ \\
\cline { 2 - 4 } & Females & $1.22 \pm 1.782$ & $0.09 \pm .503$ \\
\hline \multirow{3}{*}{ Grade level } & Grade 1-4 & $1.26 \pm 1.996$ & $0.30 \pm 0.886$ \\
\cline { 2 - 4 } & Grade 5-8 & $0.85 \pm 1.146$ & $0.00 \pm 0.000$ \\
\cline { 2 - 4 } & Grade 9-12 & $1.90 \pm 1.917$ & $0.00 \pm 0.000$ \\
\hline
\end{tabular}

the mean DMFT score was 1.77 in hearing impaired and blind young adults. ${ }^{25}$ Tahani and Heidary in Iran documented a mean DMFT score of $3.8 \pm 4.4$ for $4-12$ school students. ${ }^{43}$ However, a lower result was reported among institutionalized hearing impaired children in India $(0.37 \pm 0.88) .{ }^{44}$ The difference might be due to the difference in lifestyle and oral hygiene practice between the studies. Our findings show that decay is a major component of the mean DMFT with a mean value of $0.77 \pm 1.258$, and there is no filled tooth. This showed oral health care service is still a challenge for the hearing-impaired students in the study area.

Moreover, the present study found that sex, age, residence, sugary food intake, and tooth brushing habit did not have a statistically significant association with dental caries. A similar finding was reported in Rwanda, where gender, residency, and toothpaste use have no significant association with dental caries among children with disabilities. ${ }^{34}$ On the contrary, studies done in the USA, ${ }^{45}$ Rwanda, $^{34}$ and Tanzania ${ }^{46}$ found a statistically significant association between sugar intake and dental caries. This might be due to the difference in frequency of sugar-rich staff intake.

Our study found a statistically significant association between students' grade level, paternal educational status, dental caries, gingival bleeding on probing with periodontal disease. Subsequently, being grades 1 to 4 increased the prevalence of periodontal disease among hearing-impaired special need students by four-folds as compared with grade 9-12 students. This might be due to a low level of awareness about oral hygiene practice in lower-grade students.

In addition, parental educational status was significantly associated with periodontitis, in such a way that students whose fathers had no formal education were fivefold times more likely to have the periodontal disease compared with those who had formal education. On the contrary, grade 9-12 students were 4.45 times more likely to have dental caries than grade 1-4 students. The high prevalence of dental caries among grade 9-12 students could be attributed to the fact that the prevalence of dental caries increases with age, ${ }^{47}$ and the long-term effect of the disability might compromise their oral health status.

In addition, students with occasional parental support during tooth brushing were 4.84 times more likely to have dental caries than those who had frequent support from their parents. A study done in North Showa, Ethiopia, reported that lack of parental support during tooth brushing was significantly associated with dental caries. ${ }^{48}$ Besides, a cross-sectional study conducted on 415 preschool children and their caregivers from Curitiba, Brazil, reported a high prevalence of dental caries among special need children whose caregivers had a poor oral health knowledge (PR $=1.35$; 95\% CI: 1.01-1.79), and those with infrequent parental support during tooth brushing ( $\mathrm{PR}=1.48 ; 95 \% \mathrm{CI}: 1.16-1.91) .^{32}$ Literature indicates that low parents' or caregivers' knowledge of oral health or poor attitude has been associated with frequent use of sweet foods, bottle feeding during the night, and failure to brush their children due to negligence and frustration. ${ }^{49,50}$ 
Table 6 Factors Associated with the Periodontal Disease Among Special Need Students in Amhara Region, Ethiopia, 202। $(\mathrm{n}=149)$

\begin{tabular}{|c|c|c|c|c|}
\hline \multicolumn{2}{|l|}{ Independent Variables } & \multirow{2}{*}{$\frac{\text { COR }(95 \% \mathrm{CI})}{10.71(1.21,94.96)}$} & \multirow{2}{*}{$\begin{array}{l}\text { AOR }(95 \% \mathrm{Cl}) \\
5.02(0.47,53.80)\end{array}$} & \multirow{2}{*}{$\frac{\text { P-value }}{0.183}$} \\
\hline Age & $7-12$ years & & & \\
\hline & $13-18$ years & I. $14(0.43,3.06)$ & $0.87(0.28,2.69)$ & 0.815 \\
\hline & Above 18 years & I & I & \\
\hline \multirow[t]{2}{*}{ Sex } & Male & $0.59(0.27,1.28)$ & $0.515(0.227,1.172)$ & 0.230 \\
\hline & Female & 1 & I & \\
\hline \multirow[t]{3}{*}{ Grade level } & $1-4$ & $3.85(1.14,12.93)$ & $3.94(1.16,13.38)$ & 0.001 \\
\hline & $5-8$ & I. $.40(0.49,4.02)$ & $1.39(0.48,4.02)$ & 0.586 \\
\hline & $9-12$ & 1 & I & \\
\hline \multirow[t]{2}{*}{ Sugary food intake } & Yes & $2.69(0.57,12.64)$ & $2.99(0.59,14.97)$ & 0.184 \\
\hline & No & I & I & \\
\hline \multirow{3}{*}{$\begin{array}{l}\text { Parents support during tooth } \\
\text { brushing }\end{array}$} & Never & $0.60(0.07,5.42)$ & $1.63(0.14,18.64)$ & 0.694 \\
\hline & Sometimes & $1.40(0.12,16.46)$ & $1.92(0.15,24.4 I)$ & 0.615 \\
\hline & Always & I & I & \\
\hline \multirow[t]{2}{*}{ Comorbidity } & Yes & $2.02(0.89,4.56)$ & $1.19(0.07,19.77)$ & 0.905 \\
\hline & No & 1 & I & \\
\hline \multirow[t]{3}{*}{ Fathers educational status } & No formal education & $3.06(1.01,9.21)$ & $4.98(1.00,24.63)$ & 0.049 \\
\hline & Read and Write & $1.36(0.48,3.87)$ & $2.78(0.60,12.89)$ & 0.190 \\
\hline & Formal education & I & I & \\
\hline \multirow[t]{2}{*}{ Class-2 malocclusion } & Yes & $0.28(0.11,0.72)$ & $0.4 \mathrm{I}(0.14,1.24)$ & 0.116 \\
\hline & No & 1 & $\mathrm{I}$ & \\
\hline \multirow[t]{2}{*}{ Dental caries } & Ye & $2.47(1.13,5.38)$ & $2.5 \mathrm{I}(1.08,5.08)$ & 0.032 \\
\hline & No & 1 & I & \\
\hline \multirow[t]{2}{*}{ Medication } & Yes & $1.09(0.29,4.17)$ & $0.66(0.04$, II.50) & 0.772 \\
\hline & No & 1 & 1 & \\
\hline \multirow[t]{2}{*}{ Mouth breather } & Yes & $3.34(1.29,8.70)$ & $2.21(0.69,7.09)$ & 0.183 \\
\hline & No & 1 & I & \\
\hline \multirow[t]{2}{*}{ Bleeding on probing } & Yes & $0.10(0.04,0.27)$ & $9.892(3.687,26.537)$ & $<0.001$ \\
\hline & No & I & I & \\
\hline Years lived with disability & 0.89 & $0.89(0.80,0.99)$ & $0.96(0.85,1.08)$ & 0.465 \\
\hline
\end{tabular}

Abbreviations: $\mathrm{COR}$, crude odds ratio; $\mathrm{AOD}$, adjusted odds ratio; $\mathrm{Cl}$, confidence interval.

\section{Strength and Limitation of the Study}

This is the first study on the oral health status of hearing-impaired school students in Ethiopia. The investigators faced some challenges during data collection. The first challenge was the COVID-19 pandemic. Due to this pandemic, some hearing-impaired students withdraw from their education due to a lack of support from their friends and the community, 
Table 7 Factors Associated with Dental Caries Among Special Need Students in Amhara Region, Ethiopia, 202I ( $\mathrm{n}=149)$

\begin{tabular}{|c|c|c|c|c|}
\hline \multicolumn{2}{|l|}{ Independent Variables } & \multirow{2}{*}{$\frac{\text { COR }(95 \% \mathrm{Cl})}{\mathrm{I}}$} & \multirow{2}{*}{$\frac{\text { AOR }(95 \% \mathrm{Cl})}{\mathrm{I}}$} & \multirow[t]{2}{*}{ P-value } \\
\hline Grade level & $1-4$ & & & \\
\hline & $5-8$ & $0.91(0.44,1.88)$ & $1.29(0.529,3.15)$ & 0.575 \\
\hline & $9-12$ & $2.57(0.91,7.30)$ & $4.45(1.10,21.28)$ & 0.037 \\
\hline \multirow{3}{*}{$\begin{array}{l}\text { Parents support during } \\
\text { tooth brushing }\end{array}$} & Yes & I & 1 & \\
\hline & No & $3.21(1.36,7.57)$ & $5.10(1.89,13.76)$ & 0.001 \\
\hline & & $2.14(0.41,11.29)$ & $3.72(0.66,20.84)$ & 0.135 \\
\hline \multirow[t]{2}{*}{ Comorbidity } & Yes & $3.32(1.15,9.54)$ & $3.12(0.89,10.89)$ & 0.075 \\
\hline & No & I & 1 & \\
\hline \multirow[t]{3}{*}{ Oral health status } & Good & 1 & 1 & \\
\hline & Fair & $1.29(0.55,3.08)$ & I.3I $(0.49,3.46)$ & 587 \\
\hline & Poor & $4.60(1.98,10.73)$ & $8.07(2.49,26.18)$ & 0.001 \\
\hline \multirow[t]{2}{*}{ Class-2 malocclusion } & Yes & I.70 $(0.69,4.23)$ & $0.83(0.2 \mathrm{I}, 3.3 \mathrm{I})$ & 0.796 \\
\hline & No & I & I & \\
\hline \multirow[t]{2}{*}{ Sucking habits } & Yes & $2.09(0.89,4.91)$ & $1.52(0.54,4.27)$ & 0.424 \\
\hline & No & I & 1 & \\
\hline \multirow[t]{2}{*}{ Periodontal disease } & Ye & $2.47(1.13,5.38)$ & $0.83(0.29,2.38)$ & 0.726 \\
\hline & No & I & 1 & \\
\hline \multirow[t]{2}{*}{ Medication } & Yes & $6.87(1.83,25.82)$ & $9.26(2.17,39.45)$ & 0.003 \\
\hline & No & I & 1 & \\
\hline
\end{tabular}

Abbreviations: $\mathrm{COR}$, crude odds ratio; $\mathrm{AOD}$, adjusted odds ratio; $\mathrm{Cl}$, confidence interval.

as social distancing and face masking become mandatory. Due to the impairment, some of the students did not express their problems confidentially, and the nature of the self-reported oral health behavior has its limitation. Finally, due to the nature of the cross-sectional study, a conclusion about the causal relationship between the independent factors and the dependent factors cannot be drawn.

Despite these challenges, the current study, which was carried out extensively by multi-professionals, was successful in determining the prevalence of periodontal disease and dental caries among hearing-impaired students attending special need schools in the Amhara region, Ethiopia.

\section{Conclusion and Recommendation}

In the present study, a significant amount of special needs school students had periodontal disease and dental caries. School oral health programs and caregivers assisted oral hygiene practice is essential to combat oral health problems in hearing-impaired students. Moreover, a nationwide prospective study with a large sample size will be required to reflect the oral health status of hearing-impaired individuals in the country.

\section{Abbreviations}

DMFT, Decayed, Missed, and filled permanent teeth; dmft, decayed, missed, and filled primary teeth; SD, standard deviation; WHO, World Health Organization; COR, crude odds ratio; AOR, Adjusted odds ratio. 


\section{Data Sharing Statement}

All the data used for this study are available on request from the corresponding author (teferaden@gmail.com).

\section{Ethics Approval and Consent to Participate}

Ethical approval was obtained from the University of Gondar, institutional review board (Ref. No: V/P/RCS/05/541/ 2020). All research participants, parents, and legal guardians were properly informed about the nature of the study and the benefits of taking part in it. Before the study began, parents/legal guardians and special need schools provided written approval. The study was done in accordance with the declaration of Helsinki (Code of Ethics of the World Medical Association).

\section{Consent for Publication}

Consent for publication was obtained by all authors.

\section{Acknowledgments}

We are thankful to the research, community service, and technology transfer V/president of the University of Gondar, the research director of the College of Medicine and Health Science for providing financial support for this study, and the IRB for providing ethical approval. Furthermore, we would like to thank our outstanding data collectors and study participants, without whom this study would not have been possible.

\section{Author Contributions}

All authors made substantial contributions to conception and design, acquisition of data, or analysis and interpretation of data; took part in drafting the article or revising it critically for important intellectual content; gave final approval for the version to be published, and agreed to be accountable for all aspects of the work.

\section{Funding}

The research, community service, and technology transfer V/President office at the University of Gondar funded this work. The funding institution was not involved in the study design, data collection, analysis, or interpretation of the data, article writing, or decision on the submission.

\section{Disclosure}

The authors report no conflicts of interest in this work.

\section{References}

1. Mehra S, Eavey RD, Keamy DG Jr. The epidemiology of hearing impairment in the United States: newborns, children, and adolescents. Otolaryngol Head Neck Surg. 2009;140(4):461-472. doi:10.1016/j.otohns.2008.12.022

2. Olusanya BO, Neumann KJ, Saunders JE. The global burden of disabling hearing impairment: a call to action. Bull World Health Organ. 2014;92 (5):367-373. doi:10.2471/BLT.13.128728

3. World Health Organization. WHO global estimates on prevalence of hearing loss; 2012.

4. Fitaw Y, Boersma JM. Prevalence and impact of disability in north-western Ethiopia. Disabil Rehabil. 2006;28(15):949-953. doi:10.1080/ 09638280500404552

5. Aruna CN, Chandu GN, Shafiulla MD. Dental caries experience among deaf and dumb children in Davangere, Karnataka. J Indian Assoc. 2005;5 (6): 1.

6. Nahar SG, Hossain MA, Howlader MBU, Ahmed A. Oral health status of disabled children. Bangladesh Med Res Counc Bull. 2010;36(2):61-63. doi:10.3329/bmrcb.v36i2.6989

7. Kamatchy KR, Joseph J, Krishnan CG. Dental caries prevalence and experience among the group of institutionalized hearing impaired individuals in Pondicherry-a descriptive study. Indian J Dent Res. 2003;14(1):29-32.

8. Ajami BA, Shabzendedar M, Rezay YA, Asgary M. Dental treatment needs of children with disabilities. J Dent Res Dent Clin Dent Prospects. 2007;1(2):93-98. doi:10.5681/joddd.2007.016

9. Swallow JN. The dental problems of handicapped children. $R$ Soc Health J. 1965;85(3):152-157. doi:10.1177/146642406508500311

10. Kumar S, Dagli RJ, Mathur A, Jain M, Duraiswamy P, Kulkarni S. Oral hygiene status in relation to sociodemographic factors of children and adults who are hearing impaired, attending a special school. Spec Care Dent. 2008;28(6):258-264. 
11. Sandeep V, Kumar M, Vinay C, Chandrasekhar R, Jyostna P. Oral health status and treatment needs of hearing impaired children attending a special school in Bhimavaram, India. Indian J Dent Res. 2016;27(1):73-77. doi:10.4103/0970-9290.179835

12. Azfar M, Khan I, Quershi S, Zia N, Abid K. Oral Health Status Among Hearing and Speech Impaired Children of Karachi, Pakistan. JPDA. 2008;27:181-185.

13. Gabre P, Martinsson T, Gahnberg L. Incidence of, and reasons for, tooth mortality among mentally retarded adults during a 10-year period. Acta Odontol Scand. 1999;57(1):55-61. doi:10.1080/000163599429110

14. World Health Organization. Oral health surveys: basic methods. World Health Organization; 2013.

15. Ngom PI, Diagne F, Aïdara-Tamba AW, Sene A. Relationship between orthodontic anomalies and masticatory function in adults. Am J Orthod Dentofacial Orthop. 2007;131(2):216-222. doi:10.1016/j.ajodo.2005.03.027

16. Tefera A, Bekele B. Periodontal disease status and associated risk factors in patients attending a tertiary hospital in Northwest Ethiopia. Clin Cosmet Investig Dent. 2020;12:485. doi:10.2147/CCIDE.S282727

17. Ayele FA, Taye BW, Ayele TA, Gelaye KA. Predictors of dental caries among children 7-14 years old in Northwest Ethiopia: a community based cross-sectional study. BMC Oral Health. 2013;13(1):7. doi:10.1186/1472-6831-13-7

18. Aynalem YA, Alamirew G, Shiferaw WS. Magnitude of dental caries and its associated factors among governmental primary school children in Debre Berhan Town, North-East Ethiopia. Pediatr Health Med Ther. 2020;11:225. doi:10.2147/PHMT.S259813

19. Mulu W, Demilie T, Yimer M, Meshesha K, Abera B. Dental caries and associated factors among primary school children in Bahir Dar city: a cross-sectional study. BMC Res Notes. 2014;7(1):949. doi:10.1186/1756-0500-7-949

20. Tefera AT, Girma B, Adane A, et al. Dental health problems and treatment-seeking behavior among special need school students in Amhara region, Ethiopia. BMC Oral Health. 2021;21(1):489. doi:10.1186/s12903-021-01856-x

21. Greene JG, Vermillion JR. The simplified oral hygiene index. J Am Dent Assoc. 1964;68(1):7-13. doi:10.14219/jada.archive.1964.0034

22. Gallagher J, Scambler S. Disability and oral health. In: Learning Disabilities. 2012:343-364.

23. Hennequin M, Moysan V, Jourdan D, Dorin M, Nicolas E. Inequalities in oral health for children with disabilities: a French national survey in special schools. PLoS One. 2008;3(6):e2564. doi:10.1371/journal.pone.0002564

24. Clerehugh V, Kindelan S. Guidelines for periodontal screening and management of children and adolescents under 18 years of age. $\mathrm{Br} S o c$ Periodontol Br Soc Pediatr Dent. 2012;1:1-25.

25. Jain M, Bharadwaj SP, Kaira LS, et al. Oral health status and treatment need among institutionalised hearing-impaired and blind children and young adults in Udaipur, India. A comparative study. Oral Health Dent Manag. 2013;12(1):41-49.

26. Ameer N, Palaparthi R, Neerudu M, Palakuru SK, Singam HR, Durvasula S. Oral hygiene and periodontal status of teenagers with special needs in the district of Nalgonda, India. J Indian Soc Periodontol. 2012;16(3):421. doi:10.4103/0972-124X.100923

27. Pieper K, Dirks B, Kessler P. Caries, oral hygiene and periodontal disease in handicapped adults. Community Dent Oral Epidemiol. 1986;14(1):2830. doi:10.1111/j.1600-0528.1986.tb01489.x

28. Rajabloo S, Pakkhesal M, Naghavi Alhosseini A, Ghorbani Z, Rajabi A. Mothers view about oral health status, oral hygiene behaviors, and dental services utilization of their children with hearing impairment attending special schools. Spec Care Dentist. 2021. doi:10.1111/scd.12648

29. Pareek S, Nagaraj A, Yousuf A, Ganta S, Atri M, Singh K. Effectiveness of supervised oral health maintenance in hearing impaired and mute children-A parallel randomized controlled trial. J Int Soc Prev Commun Dent. 2015;5(3):176. doi:10.4103/2231-0762.159953

30. Shyama M, Al-Mutawa SA, Morris RE, Sugathan T. Dental caries experience of disabled children and young adults. Community Dent Health. 2001;18(3):181-186.

31. Al-Qahtani C, Wyne AH. Caries experience and oral hygiene status of blind, deaf and mentally retarded female children in Riyadh, Saudi Arabia. Trop Dent J. 2004;27:37-40.

32. Montes GR, Bonotto DV, Ferreira FM, Menezes JVNB, Fraiz FC. Caregiver's oral health literacy is associated with prevalence of untreated dental caries in preschool children. Cien Saude Colet. 2019;24(7):2737-2744. doi:10.1590/1413-81232018247.18752017

33. Rawlani S, Rawlani S, Motwani M, Bhowte R, Baheti R, Shivkuma S. Oral health status of deaf and mute children attending special school in Anand-Wan, Warora, India. J Korean Dent Sci. 2010;3(2):20-25.

34. Uwayezu D, Gatarayiha A, Nzayirambaho M. Prevalence of dental caries and associated risk factors in children living with disabilities in Rwanda: a cross-sectional study. Pan Afr Med J. 2020;36:193.

35. Singh A, Agarwal A, Aeran H, Dhawan P. Oral Health \& Quality of Life in preadolescents with hearing impairment in Uttarakhand, India. $J$ Oral Biol Craniofac Res. 2019;9(2):161-165.

36. Vichayanrat T, Kositpumivate W. Oral health conditions and behaviors among hearing impaired and normal hearing college students at Ratchasuda College, Nakhon Pathom, Thailand. Southeast Asian J Trop Med Public Health. 2014;45(5):1228.

37. Wei H, Wang Y-L, Cong X-N, Tang W-Q, Wei P-M. Survey and analysis of dental caries in students at a deaf-mute high school. Res Dev Disabil. 2012;33(4):1279-1286.

38. Alkahtani FH, Baseer MA, Ingle NA, et al. Oral health status, treatment needs and oral health related quality of life among hearing impaired adults in Riyadh City, Saudi Arabia. J Contemp Dent Pract. 2019;20(6):744.

39. Diéguez-Pérez M, de Nova-garcía M-J, Mourelle-Martínez MR, Bartolomé-Villar B. Oral health in children with physical (Cerebral Palsy) and intellectual (Down Syndrome) disabilities: systematic review I. J Clin Exp Dent. 2016;8(3):e337. doi:10.4317/jced.52922

40. Hamad AM, Ali SH, Arif AN, Zardawi FM. Oral health status of deafness persons in Sulaimani city-Iraq. IOSR J Dent Med Sci. 2015;14 (9):69-73.

41. Oredugba FA, Akindayomi Y. Oral health status and treatment needs of children and young adults attending a day centre for individuals with special health care needs. BMC Oral Health. 2008;8(1):30. doi:10.1186/1472-6831-8-30

42. Altun C, Guven G, Akgun OM, Akkurt MD, Basak F, Akbulut E. Oral health status of disabled individuals attending special schools. Eur J Dent. 2010;4(4):361.

43. Tahani B, Heidary A. Assessment of oral hygiene and oral health status of 4-12 year old children with hearing impairment. J Mashhad Dent Sch. 2016;40(1):59-72.

44. Jnaneswar A, Subramaniya GB, Pathi J, Jha K, Suresan V, Kumar G. Assessment of dental caries and periodontal status in institutionalized hearing impaired children in Khordha District of Odisha. J Indian Soc Pedod Prev Dent. 2017;35(3):203. doi:10.4103/JISPPD.JISPPD_11_17 
45. Ismail AI, Lim S, Sohn W, Willem JM. Determinants of early childhood caries in low-income African American young children. Pediatr Dent. 2008;30(4):289-296.

46. Rwakatema DS, Ng'ang'a PM. Early childhood caries in Moshi, Tanzania. East Afr Med J. 2010;87(7):304-310.

47. Cheng Y-H, Liao Y, Chen D-Y, Wang Y, Wu Y. Prevalence of dental caries and its association with body mass index among school-age children in Shenzhen, China. BMC Oral Health. 2019;19(1):270. doi:10.1186/s12903-019-0950-y

48. Shitie A, Addis R, Tilahun A, Negash W. Prevalence of dental caries and its associated factors among primary school children in Ethiopia. Int $J$ Dent. 2021;2021. doi:10.1155/2021/6637196

49. Vann JWF, Lee JY, Baker D, Divaris K. Oral health literacy among female caregivers: impact on oral health outcomes in early childhood. $J$ Dent Res. 2010;89(12):1395-1400. doi:10.1177/0022034510379601

50. Sanzone LA, Lee JY, Divaris K, DeWalt DA, Baker AD, Vann Jr WF Jr. A cross sectional study examining social desirability bias in caregiver reporting of children's oral health behaviors. BMC Oral Health. 2013;13(1):1-9. doi:10.1186/1472-6831-13-24

Clinical, Cosmetic and Investigational Dentistry

\section{Publish your work in this journal}

Clinical, Cosmetic and Investigational Dentistry is an international, peer-reviewed, open access, online journal focusing on the latest clinical and experimental research in dentistry with specific emphasis on cosmetic interventions. Innovative developments in dental materials, techniques and devices that improve outcomes and patient satisfaction and preference will be highlighted. The manuscript management system is completely online and includes a very quick and fair peer-review system, which is all easy to use. Visit http://www.dovepress.com/ testimonials.php to read real quotes from published authors.

Submit your manuscript here: https://www.dovepress.com/clinical-cosmetic-and-investigational-dentistry-journal 